\title{
Re-defining the Role of Interactive Architecture in Social Relationships
}

\author{
Kristina Boychenko \\ School of Architecture, University of Queensland \\ Brisbane, Australia \\ k.boychenko@uqconnect.edu.au
}

\begin{abstract}
With rapid advance of new technologies and mediated built space has shifted from a static context of functions serving users to a new participant of social relationships. Interactive abilities and computational power allow built space to become smart, dynamic, and interactive, gaining agency, able to receive information and think, perceive and learn, respond and change behavior in real time. This paper considers architectural components and users as participants of a social network and investigates their agency within this network, modes of interaction and how the components of this system influence each other.

Perception of space within or outside of the building body has become a derivative of interaction between the space and the users, and therefore subject to design and programming by architects. The principal goal of this paper is to investigate the new definition of social role of interactive architecture and explain how it communicates with users, investigate the new properties it has and how does it influence users' behavior and space awareness. It reveals the importance of bi-directional communication between society and interactive environment.

Interactive space works as a mirror, reflecting social and cultural context, or a double-sided mirror allowing interactive environment to observe users and decide how to act in accordance with these observations. Within the framework of this discourse, architectural components and people are treated as agents of one socio-technical network with equal rights and agency. It considers both human and non-human elements equally as actors within a network, employing the same analytical and descriptive methodology to all actors within a heterogeneous network.
\end{abstract}

Keywords: Interactive; Socio-technical; Agency; Network; Communication; Monad

\section{Introduction}

Motion and information are two fundamental terms of contemporary lifestyle. It gets more apparent day by day due to rapid advance of databases and the urge of society to have fast and easy access to information and getting used to surround ourselves with smart and evolving on daily basis devices in a well-ordered space customized. This accelerated development of technologies causes dramatic escalation of demands they have to satisfy and issues they aim to solve. In the age of Internet of Things, society seeks to obtain answers to requests that haven't been made yet. Society aims at total consciousness about the world around us and at a continuous dialogue between people and other actors, sending, receiving and mediating information. Conventional built space without interactive properties is restricted in its capacity to interact with varying conditions and with the people inhabiting this space. This space is static and has only one pre-defined way of being, while thanks to technological progress it can be flowing, shifting, changeable, and interacting with its users and society. Developed technologies offer multiple possibilities for information exchange between users and built environment. This new mediated environment gains self-awareness and learns how to improve its own qualities. By comprehending itself, interactive space learns how to understand its inhabitants, therefore helping them to gain self- and social awareness, too. (Figure 1) 
As Lloyd (1998) put it, based on the words of Louis Sullivan, form should not just follow function but become one with it. This can be understood in the way that the form should not be barely a response to the function, but rather be inviting, immersive, narrating about the activity performed inside. It should answer the users' question before it was even formulated, foreseeing it and communicating the right information.

As a contemporary phenomenon, interactive architecture heavily relies on expansion of new technologies. This type of digitally mediated space has tuned into a data flow, asserting its constant transformation, offering the qualities exchange between the space and the user, society and technology. As it has been proposed by Merleau-Ponty (2017), interactive space obliterates the difference between the context and the user. The actor (or the user) is introduced by the ability to move and perceive.

Contemporary society tends to erase the boundaries between static and moving, still and reactive. New technologies allow to reduce the boundaries between the user and the space, inside and outside, virtual and real, allowing existence of the real, virtual and augmented spaces at the same time. The urban development also started to consider not only tangible, but also virtual activities.

With this in mind, the question raises: how do these opposite concepts merge? What forms the definition of the place and user or its physical representation in the world of changeability and uncertainty? How can architectural environment position itself, have identity and communicate with the world and other environments? Static identities, as well as unresponsive ones, can no longer satisfy social needs in contemporary technology-driven world. This presents the revolution of the tangible and perceptive spaces, that no can serve as medium of information, interacting with each other and other spaces and/ or users, virtual and real, close or remote. All the actors of these interactions are considered as nomads, aimed at information exchange, wandering and fluctuating continuously and remaining uncertain, generating unique scenarios and communications (Mahdalickova, 2009).

By means of changing various features, including light, sound, motion and changing its components, configuration in space interactive built environment submerges the user into the atmosphere shaped for specific activities performed there, translating information and presenting it to the user in its final form. The user gets engaged in a dialogue with interactive space, following the invitation created by the space, discovering information, hidden or exposed modes of its operation, new activities, social communications, playful activities, fun situation and references that the visitors tend to interpret as the way their culture, intelligence, mood or other factors allow them to.

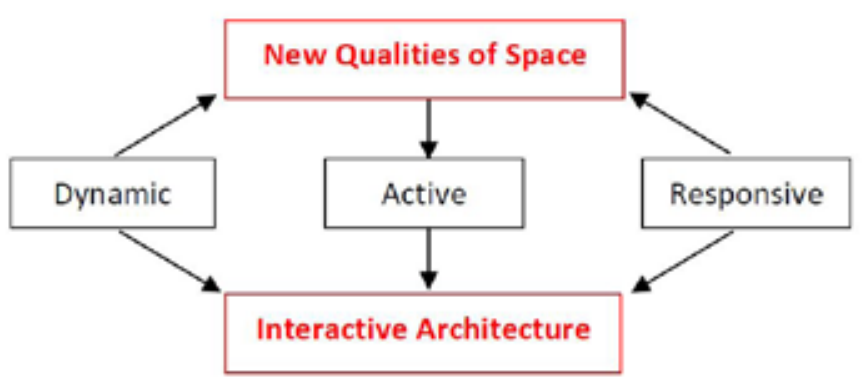

Figure 01:. New qualities of interactive space scheme.

\section{Emerging Typology of the Space}

The social conditions remain subject to alteration with fast developing digitally facilitated environment together with emergence of new types of space. These new space types share similar universally 
applicable qualities and can be eventually considered as one new space typology, able to accommodate various functions. This shift has happened due to entering the era of cyber space and dematerialization of the world around us.

This emerged type of space needs to be mobile and adjustable so that it can successfully satisfy new and constantly changing needs of society, including social and psychological demands.

This new space type heavily relies on technological advancement to define new ways of technologies and their application, allowing interactive behavior involving reaction in real time and dynamic spatial adjustment.

Interactive space behavior is based on the idea of bi-directional communication that involves both participants to be actively engaged in it, not merely the ability to adopt. Indeed, communication between two actors of society is interactive; they both listen [input], think [process] and talk [output] (Figure 2). Interactive space introduces the art of building relationships and communication of its components between each other and the communication of these components with the users. Finally, interactive built environment shapes communication between people, communicates with society and benefits it on multiple levels (Ishii \& Brygg, 1997).

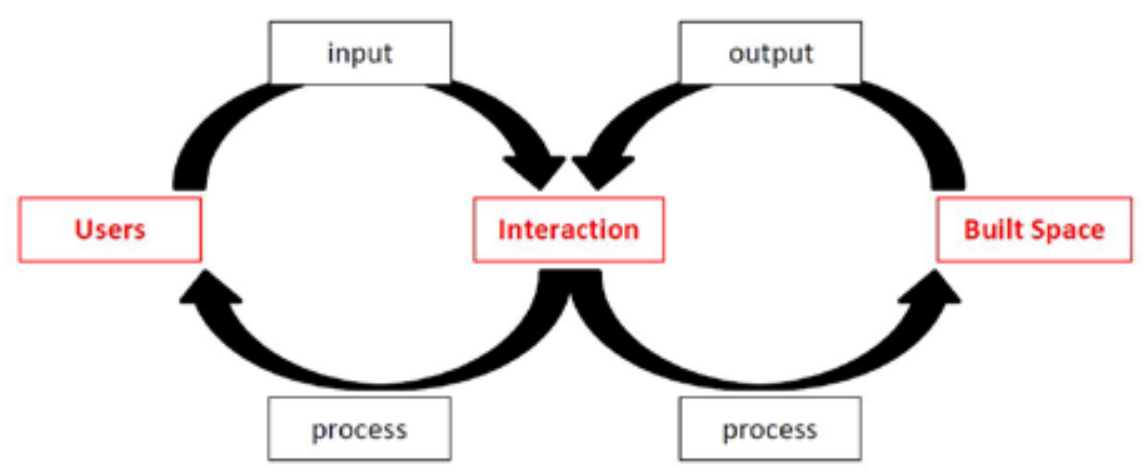

Figure 02: Concept of bi-directional communication.

Architectural space arranges social processes of interaction and plays a role in the formation and balance of social order. Architectural performance includes ownership, spatial exclusion and distinction by means of tangible boundaries with corresponding rights of access. Built space organizes social situations and provides coordination for the participants of social processes thus arranged, allowing them to find the right place of their own accord. Therefore, architectural environment reflects social organization.

A correctly located and communicated space represents an interaction, specifically an offer to take part in a particular interaction activity that is subject to acceptance or rejection by the person. In case if the offer is accepted, communicated by a user stepping into the space, the space performs as a shared ground of all members of interactive activity and accommodates all future interactions that subsequently occur within it. Merging with other design disciplines such as urban design, interaction design, sociology, psychology and many more, the space creates a cohesive narrative, an active system with broadly applicable qualities of physiognomy represented by its spatial components and the image of the built space as such (Oosterhuis, 2003).

Thanks to rapid technological advance, architectural space shifted from being a silent witness of the activities performed within it to an active participant. Computational power allowed the space to be 
smart, gaining new intellectual and intelligence qualities with development of the new technologies, obtaining interactive properties and eventually its own agency. The space is not limited to adaptation to various conditions but actually becomes the receiver, the processor and the translator of data, reacting to the new conditions and learning to communicate and act. The concept of spatial agency relies on translation of received information, like visitors' motion, location or voice commands, information about the environmental conditions or other factors. Therefore, by communication with society, interactive space becomes an actor in social relationships, changing and responding to other actors and changes of the context. This paper considers interactive space as an active part of social relationships through the Actor-Network theory. This way social relationships are considered as parts of common network where visitors or users (human) and space components (non-human) actors all have agency. Interactive behavior is defined as the ability of all components, human and non-human, to demonstrate agency, creating unique situations and communications in real time. (Boychenko, 2017) (Figure 3).

Built space has to refer to specific context, physical or digital. For example, the space can be linked to another space or spatial component, located elsewhere. Architecture must be linked to other environments, analogue as well as digital. Multimedia approach allows to create new types of interactions between the space and the users or another space, introducing universally distributed information exchange, a groundbreaking extension of the space into the new dimension. The space has become active and able to offer not just a set of structural components and functions but creating dynamic situations.

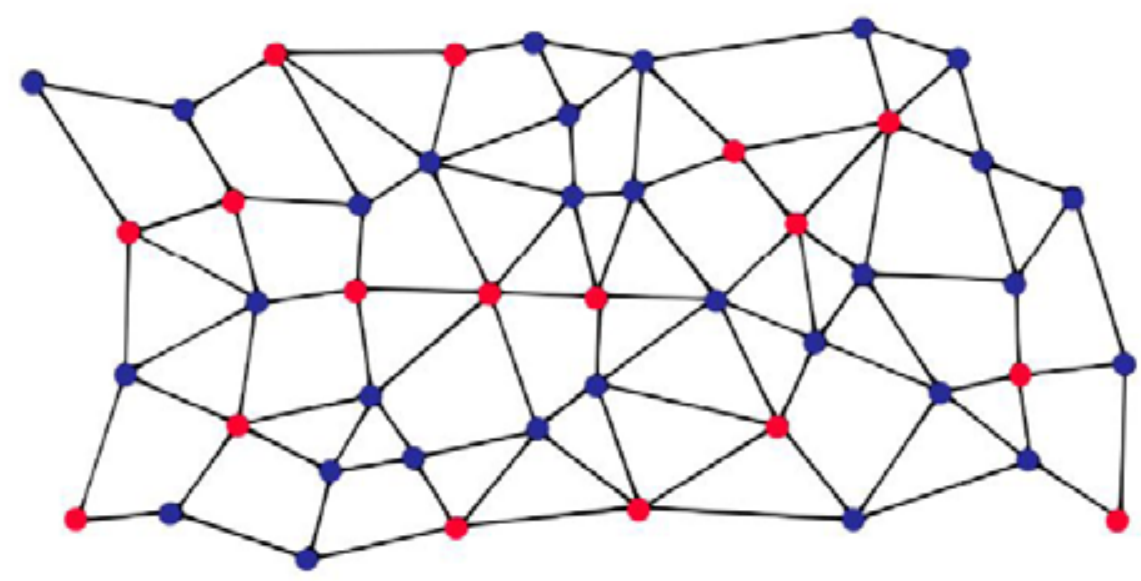

Hunan actors of the network

Non-human actors of the network

Figure 03: Human and non-human components within a homogeneous network.

Connected to other systems, interactive space can become reprogrammable, thus adaptable to various emerging conditions and situations. This unique ability to stay updated leads to adjustability of the space for different and changing functions, making it a crucial factor of the spatial performance in social network.

Social conditions can be reflected through architectural space in the following ways:

- shape adjustment to satisfy users' needs 
- space adaptability for various activities

- connection to remote spaces, devices or users

- space extension within the network

These new qualities allow the space to adjust to new conditions and react in order to maintain communication with society on a new level culturally, acting as a time transmission. The time that can accommodate all sorts of content. This way the space communicates not merely a function, but also information, changing through time. "The result is the rise of Digital Gothic", as Kas Oosterhuis,2019 puts it.

\section{Space Behavior and Consciousness}

Behavioral design is largely referred to as a type of design that can successfully satisfy various users' needs, as well as respond to their feelings and the way they occupy the space, showing awareness of the current social structure and the ways users communicate, adjusting to emerging users' needs. This concept considers behavioral design qualities and interaction of the space that acts as the context of the users 'activities and behavior.

Combs and Brown (2018) in their book argue that behavioral design can be executed to its full potential when it correlates with the idea of transparency, benefits social stability and is targeted at satisfaction of users' desires. While behavioral design is aimed at persuasion of users, it also must be ethically reasonable and respect users' intrinsic rights to freedom of choice, autonomy and dignity.

When the cue in the space is perceived by the users, they tend to learn to associate the cue with a certain action (behavior). By performing the action, users get rewarded for it (meeting the consequence of their action). A good behavioral can place created cues in space to cause certain behavior by users (Kong, 2018).

Since interactive architectural space has agency of its own, it certainly also has the intelligence of its own. Conscious spaces become active members of society, understanding the world around them, sharing a common goal that the machine understands its meaning, hence acquired initiative that drives its endeavor to search for the answers and ways of performing.

Intelligent spaces within the framework of this paper share common understanding of the world with users, developing knowledge and understandings of the world through interactions between them and the physical environment, responding differently to interactions with the context and other bodies (Oosterhui, 2006).

\section{Understanding Users}

Interactive space allows to create immersive and compelling experience and influence the way users perceive this space and the way they act there. There are different strategies to do that: one is to persuade people to level-up during a fun and engaging activity. Some researchers claim that it has positive impact on emotional state, productivity and creative thinking. Another strategy to achieve this effect is to create the environment addressing its function to playfulness while allowing benefiting through playful behavior.

According to Anderson (2011), there is a formula of interactive space:

Delight/ Fun + Exercise/ Achievement = Interactive/ Immersive space

The space resulting from this formula focuses on interactive space behavior as a tool, allowing to feel engaged, actively present in the moment, focused on the activity and immerse into the social and 
physical context of the moment and situation.

Interactive space is considered to be enjoyable due to immersive way of spatial experience, creating individual communication with the context and expression through play/interaction. This interactive behavior of communication with the space leads to emotional affection and attachment to the space (Andeson, 2011).

\section{Learning from Users}

There are various ways of influencing users' behavior through spatial means. First of all the space is required to learn how to understand the users. By utilizing various learning mechanisms, for instance distributed adaptive control, intelligent space explores effective cues that can be perceived by the users and followed by a certain behavior. By understanding users, interactive space applies bi-directional symbiotic method to communicate between the users and the space, allowing them to perceive and affect each other.

People often demonstrate the desire to learn new behaviors naturally if they are well motivated. At the same time, interactive space behavior heavily relies on the users' performance or other factors. Interactive space can communicate with users via the cues about the activity within it, the direction of movement, possible ways of using spatial components, other users etc. Smart space often has the ability to track users and analyze their willingness to follow the cues it provides. This way the space learns what cues are effective and adjusts the way it communicates.

While use of voice-based commands and gestures cannot be applied universally and can cause misunderstandings in different social groups, the communication between the users and the space can be successfully achieved by using non-verbal language of space alteration. The space can effectively use light, sound and motion as output and tracking, cameras and sensors as input for communication. Unlike spoken or written words of language-based communication, non-verbal means of communication allow to avoid antropomorphization of the spatial components.

Within the context of this paper, talking about smart spaces refers to space perception as the main feature of communication, introducing the intelligence that is now extended in its efficacy by the sensibilities of affect. This means that the users' communication with smart spaces relates to, arises from, or deliberately influences emotions.

\section{4. [E]motive Design}

According to Picard (1997), emotive design refers to affective. Effective rationality requires emotion as one of its' major components. Affective computing constitutes some shared hypothesis. First, affect implies a particular domain of cognition that can be addressed through analysis of its components. Affect is introduced as an expression of an underlying emotional state. Suchman (2007) argues that affective communication can be achieved through the "replication of behaviors understood to comprise it, made up of units assembled into a catalogue of affective expressions, productions, recognitions, and normative responses." [E]motive states of design and their affective expression can be considered as a kind of primal but still functional ancestor of contemporary reason.

\section{Pragmatic vs Humanistic}

Pragmatic applications of interactive architecture are targeted on solving issues, satisfying demands and systems optimization. It suggests numerous improvements in comparison to static architecture like the enhancement of the building's ability to meet functional demands, its security, flexibility and adaptability, image, reliability, energy consumption, expenses and cost, and easiness in using it; optimization of visual, acoustic, thermal and other conditions, meeting the changing requirements in 
public and private space, accommodating special needs and desires. It is the answer to how architecture can help the inhabitants to perform their activities or give advice on how to use the space in more efficient, sometimes unexpected, ways, or providing them with more suitable conditions for their tasks.

The humanistic approach is aimed at analysis of the impact of interactive space communication of users' physical, psychological, and social conditions, referring to artistic implications of the space. It considers the design of dynamic spatial components linked to the way users perceive the space and behave in it, moving and shifting their vantage point. Hoberman (2007) talks about the influence of motion on emotional state: "When one sees this special behavior [of transformation], one feels it in one's body - perhaps a physiological connection, because there is a sensation, a physical sensation and a mental perceptual sensation."

Since very early in the development of interactive space design as a field, the description of a new world where buildings have evolved alongside a futuristic society to assume new functional and social roles has populated the imagination of architects. This expectation is justified by two reasons: first, because it is known that technology has the potential to catalyze profound cultural transformations; second, because interactive architecture carries the potential to cause unprecedented shift to buildings' capabilities, which have otherwise evolved at a very conservative pace.

\section{The Perspective}

The concept of spatial agency can be appreciated through a cybernetic perspective. Following the assumption that every interactive system has an internal goal, that makes feedback structures conceivable, then every single interactive system should have some degree of intrinsic agency, which does not necessarily mean complete autonomy or intelligence. Therefore, extrinsic agency of space o spatial components means that every interactive setting also has intrinsic agency (following Bruno Latour's definition).

Cybernetic background explains the initial relation between interactive space and users' agency. Being the key figure in the second-generation cybernetics, Pask (1969) addressed the importance of feedback in systems design and components specification in space design. By means of smart systems, interactive space can become active participant empowering environments by including the users as participants of a larger control loop.

According to Pask (1969), "the designer is no longer conceived as the authoritative controller of the final product" of the interactive space. On the contrary, "an environment should allow users to take a bottom up role in configuring their surroundings in a malleable way." Haque (2007) referred to Pask's approach to architecture in the following way, "it is about designing tools that people themselves may use to construct - in the widest sense of the word - their environments and as a result build their own sense of agency" (Pask, 1969).

In general, the idea of interactive space behavior often referred to the concept of user empowerment in the last century literature. It is argued that early addressing of interactive behavior in media studies sought to subvert traditional systems that helped reproduce power and social relations.

\section{Conclusion}

Interactive architecture is dynamic and time-based, meaning it changes its performance (in terms of space or in terms of behavior), demonstrating a significant shift from static and fixed. Users become parts of performance within the context of interactive environment as they do also change over time.

Both built spaces, space components and users can be considered as actors or small particles within 
one homogeneous network, constantly perceiving each other and changing accordingly; monads within a system, aiming at achieving a common goal. They demonstrate swarm behavior and spatial as well as social awareness of each other's actions, constituting a system where their roles are defined by the same set of rules. Both human and non-human participants become interchangeable monads, able to influence the system with the same or equal degree of agency in this universal decision-making process.

Within the framework of social communication these monads as the result of their interaction create unique social relationships and play equal roles in this communication, influencing behavior of other monads, changing and adjusting to them. Spaces shape the ways users behave in it and influence the ways they interact with each other and the space, while the users change the space performance, improving its behavior and creating unique customized spatial organizations.

Berkel (2009) said that the value of our architecture is to inspire the users to generate ideas and images, therefore to make it attractive to people, to make them stay longer and come back to places that the architects create for them.

Functionality and value can be found within the object and at the same time is superimposed on its cover. Architectural mediated spaces have turned into complex adaptive systems, interacting with environment, outside and from within (Kemp, 2009).

Communicative performance of public spaces incorporates social and cultural properties of space. A plethora of case studies within the discipline demonstrate taking advantage of the medium to engage society through participation. Purposefully designed, specially defined interactions are utilized to comprehend, organize and stimulate social communication. While tangible architectural environment means are utilized to accommodate functions, navigate the flow of users of the space and allow or limit their movement within the space, interactive spatial means focus on having significant effect on social interactions (Marialuisa, 2013).

Interactive components of designed space provide attachments and communication scenarios, which allow congregation of the network actors, forming various groups, assembling social diversity, linking heterogeneous elements and effects, thus adjusting and enacting the social, triggering foreseen and unforeseen situations.

Architecture that lacks interactive properties can successfully accommodate physical function and communication between people, playing the role of silent contextual background, offering shelter and non-communicative passage through it. At the same time, interactive qualities make the space a participant of social relationships, shaping it and persuading a dialogue between the society, represented by users, and the space. Smart architectural environment actively participates in unique situations and shapes experience, involving users individually and as social clusters in various activities, offering them to communicate with the space on individual or group level. Interactive environment and users get involved in a real time dialogue, stating further modes of their communication and shaping new experiences.

\section{Bibliography}

Andeson, Stephen (2011). Seductive Interaction Design: Creating Playful, Fun, and Effective User Experiences (Voices That Matter). Berkeley, CA: New Riders.

Ben van Berkel, B.V., \& Bos, C. (2006). “After Image.” (Plz identify source type)

Boychenko, Kristina (2017). Interactive Architecture: development and implementation into the built environment. European Journal of Technology and Design 
Boychenko, Kristina (2017). Senses and Behavior of Interactive. Bulletin of Science and Education.

Brown, T. Dalton Combs PhD \& Ramsay A. 2018. Digital Behavioral Design. Boundless Minds.

Frank Lloyd Wright (1998) Performed by documentary. (Plz reveal more details)

Ishii, H., \& Brygg, U. (1997). Tangible Bits: Towards Seamless Interfaces between people, bits and Atoms. ACM CHI 97 Human Factors in Computing System Conferrence March 22-27. Atlanta, Georgia: Steven Pemberton, 234-241.

Hoberman, Chuck (2007). Interview by PingMag. Transformable Architecture (July).

Kemp, M. F., \& Miles (2009). Interactive Architecture. 97-105: Princeton Architectural Press.

Kong, Joy (2018, June 29). What is behavioural design. UX Planet. Retrieved from https://uxplanet.org/what-isbehavioural-design-8d2790a9d624.

Mahdalickova, Eva (2009). New Experiences Of The Body Through Space. (Please reveal full details)

Marialuisa, Palumbo (2013). Looking at the first neuromorphic space, a conversation with Paul Verschure. Retrieved from http://architettura.supereva.com/interview/20040205/index_en.htm.

Oosterhui, Kas (2006). Swarm Architecture II. Delft: TU Delft.

Oosterhuis, Kas (2003). Hyperbodies. Toward an E-motive Architecture. Turin: Testo \& Immagine.

Pask, Gordon (1969). The Architectural relevance of Cybernetics. Architectural Design, 496.

Picard, M. W. (1997). Affective Computing No. 321 (M.I.T Media Laboratory Perceptual Computing Section Technical Report). Cambridge: M.I.T.

Suchman, Lucy (2007). Human-Machine Reconfigurations: Plans and Situated Actions, 2nd Edition. New York: Cambridge University Press.

Cite this article as: Boychenko K., "Re-defining the Role of Interactive Architecture in Social Relationships", International Conference on the $4^{\text {th }}$ Game Set and Match (GSM4Q-2019), Doha, Qatar, 6-7 February 2019, https:// doi.org/10.29117/gsm4q.2019.0016 